\title{
The Formation of Magnetite Nanoparticle in Ordered System of the Soybean Lecithin
}

\author{
Tiefu Li,' Yingiie Deng, Xiaoping Song, Zhixiong Jin, and Ying Zhang \\ Department of Pharmact, Shenvang Pharmaceutical Universin, Shemang Liaoning 1 10016, China \\ ${ }^{t}$ Institute of Metals Research. Chinese Academia of Science. Shenvang Liaoning 1 10016. China \\ Received October 19.2002
}

\begin{abstract}
A method of preparation of magnetite nanoparticles in ordered systems, as in vesicle and microenulsion, consisting of soybean lecithin and water has been introduced. The size of magnetite grain was controlled by the content of soybean lecithin and size of liposomes in the systems. It was found by experiment that magnetite nanoparticles were formed inside the double layer vesicles. The magnetite nanoparticles were separated by magnetic separation and centrifugation and the dispersion of the magnetite nanoparticles prepared at $10 \%(w / w)$ soybean lecithin was particularly stable. The formation of pure nagnetite nanoparticles was confirmed by analyses of XRD and electron diffraction pattern.
\end{abstract}

Key Words : Nanoparticles. Soỵbean lecithin. The ordered șistems. Magnetite

\section{Introduction}

Nanoparticles in the size range of nanometer have attracted great attention researchers at overseas in recent years. They exhibit unusual physical and chemical properties significantly different from those of the bulk materials due to their extremely small size and large specific surface.$^{1-4}$ Magnetic nanoparticles of spinel ferrites are of great interest in fundamental science. especially for contrast enhancement in magnetic resonance imaging (MRI) ${ }^{5-11}$ By using aqueous intravesicular precipitation to prepare nanoparticles such as $\mathrm{Al}_{-} \mathrm{O}_{3}$ has been attempted ${ }^{12}$ and also was reported to prepare magnetite in the Poly' (vinyl alcohol) (PVA). ${ }^{13}$ but there is no reported to prepare magnetite nanoparticles with soy bean lecithin in ordered systems. Because the ordered systems composed of soybean lecithin with water have nanometer space. we use them as nanoreactors to prepare magnetite nanoparticles and to observe as well as to study the size and structure of the magnetite. which is very important to apply them to such as medicine. chemistry and information technology

In the present work. we used the soybean lecithin as the nanoreactors. which was not only as a stabilizer but also a protective agent in the preparation of magnetite nanoparticles by the co-precipitation and the final product were also studied.

\section{Materials and Methods}

Materials and Instruments. Soybean lecithin (Grade Injection) is purposed from Fat Plant of Shanghai of China. Water was doubly distilled and pure nitrogen gas was bubbled through it for more than 2 lus just before use to remove the dissolved oxy'gen. Nitrogen was purified from

"Corresponding author. Tel: +86-24-23843711: Fax: +86-2423917603 ; e-mail: 1tfsybe tathotmail.com traces of carbon dioxide and oxygen by passing the gas through a basic pyrogallol solution. The other reagents are analy zed reagents.

In order to observe morphology and particle size. we used a JEM-2000EXII field emission gun transmission electron microscope operated at $200 \mathrm{kV}$ and with the vacuum better than $2 \times 10^{-6} \mathrm{~Pa}$ and the typical electron diffraction pattern was taken.

The size distribution of particles in a supernatant fluid was also determined by photon correlation spectroscopy method (PCS) (Zetasizer 3000. Malvern Instruments. GB).

$\mathrm{X}$-ray diffraction (XRD) was conducted on a Rigaku D/ max-2500 PC X-ray diffractometer using $\mathrm{CuK} \alpha$ radiation to determine the crystal structure

Adsorption of the soybean lecithin on the surface of $\mathrm{Fe}_{3} \mathrm{O}_{4}$ powders was examined by infrared spectroscopy (Hitachi model IR-440 infrared spectrometer).

Thermogravimetric analysis (TGA) was done in the nitrogen gas with a heating rate of $10^{\circ} \mathrm{C} \mathrm{min}^{-1}$ up to $480^{\circ} \mathrm{C}$ on the Perkin-Elmer instruments.

Preparation of Magnetite Nanoparticles. A mixed solution of ferrous and ferric ions in the molar ratio $1: 2$ was prepared by dissolving $0.10 \mathrm{~mol} \mathrm{FeCl}-6 \mathrm{H}_{2} \mathrm{O}$ and $0.20 \mathrm{~mol}$ $\mathrm{FeCl}_{3} \cdot 4 \mathrm{H}_{2} \mathrm{O}$ in a $10 \%(\mathrm{w} / \mathrm{w}) 100 \mathrm{~mL}$ aqueous solution of soybean lecithin at $25^{\circ} \mathrm{C}$ by sonication with ultrasonic homogenizer under nitrogen. Magnetite was precipitated by adding $50 \mathrm{~mL}$ of the above mentioned mixed solution to 100 $\mathrm{mL}$ aqueous solution of $2 \mathrm{~mol} \cdot \mathrm{L}^{-1} \mathrm{NH}_{3}(\mathrm{aq})$ at $35^{\circ} \mathrm{C}$ while stirring at $1000 \mathrm{rpm}$ using an impeller and the reaction mixture was kept at $\mathrm{pH} 8-9$. The mixture was subsequently aged at $35^{\circ} \mathrm{C}$ for $30 \mathrm{~min}$ and cooled to room temperature After the end of magnetite precipitation. $\mathrm{pH}$ decreased to 5.0. Precipitates and supernatant fluid were separated by magnetic separation and centrifugation. The precipitates were washed with deionized water under ultrasonication for $30 \mathrm{~min}$ and then separated by centrifugation at $10000 \mathrm{rpm}$ for $60 \mathrm{~min}$. The source magnetite sol of ultrafine particle was 
dialyzed through a cellophane film against rumning water until $\mathrm{pH}$ 6-7 and subsequently dried under vacuum at $35^{\circ} \mathrm{C}$ before characterization.

\section{Results and Discussion}

Morphology and Particle Size. Figure 1 shows the morphology of the samples the value for $\mathrm{Fe}_{3} \mathrm{O}_{4}$ coated soy bean lecithin is about $7 \mathrm{~nm}$ by TEM. Figure 2 shows the mean size of particles in a supernatant fluid by PCS. The average size of particles is $164.8 \mathrm{~mm}$ analyses by intensity. $163.1 \mathrm{~mm}$ by volume and $158.3 \mathrm{~nm}$ by number respectively. We can see clearly from Figure 1 and Figure 2 that the nanoparticles were well dispersed by the lecithin. The result

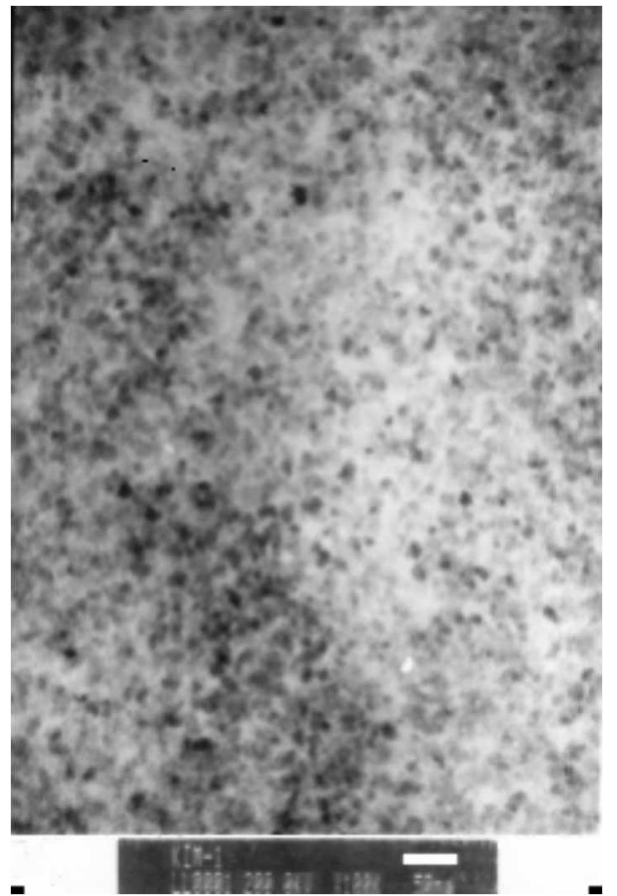

Figure 1. TEM photonicrograph of $\mathrm{Fe}_{3} \mathrm{O}_{4}$ nannoparticles $(\mathrm{Bar}=50$ nim). shows that such a fine particle size could be attributed to the inhibition effect of lecithin and also shows that the lecithin used as the stabilizer and the protective agent in the precipitation of $\mathrm{Fe}_{3} \mathrm{O}_{4}$ nanoparticles is also effective.

The result shows that the particle size of nanoparticle maybe attribute to the inhibition effect of soybean lecithin and also shows that soybean lecithin use as stabilizer and a protective agent in the precipitation of $\mathrm{Fe}_{3} \mathrm{O}_{4}$ nanoparticles is also effective. It was found by experiment that the size of $\mathrm{Fe}_{3} \mathrm{O}_{4}$ has increasing with the decrease of the concentration of soybean lecithin. The reason is probably that soybean lecithin is an amphipathic molecule. having a hydrophilic headgroup and two long hydropholic hydrocarbon chains. When their concentration exceed critical micelle concentration $\left(\mathrm{CMC}=1.6 \times 10 \mathrm{~mol} \cdot \mathrm{L}^{-1}\right.$. double layer liposomes of the ordered systems are formed. which limited the particle size of the $\mathrm{Fe}_{3} \mathrm{O}_{4}$ particles. Magnetite was formed in the double layer liposomes of the ordered systems as Figure 3. one part magnetite was encapsulated within the double layer liposomes. and another part was formed in the extravesicular. the whole solution was divided numberless micro compartment. Since the vesicle wall is permeable to the hydroxyl ions. they diffuse down their chemical potential gradient into the intravesicular space and react with the available ferrous and ferric ions to form an intravesicular product. The typical electron diffraction pattern was shown in Figure 4.

Coverage and Adsorption by Soybean Lecithin. Figure

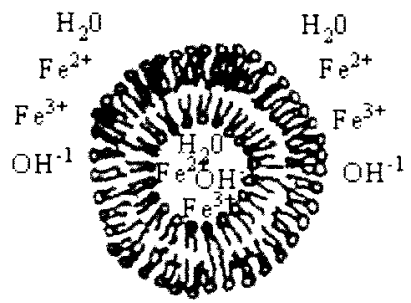

Figure 3. Formation of $\mathrm{Fe}_{3} \mathrm{O}_{4}$ nanoparticles in the double layer liposomes of the ordered ststems.
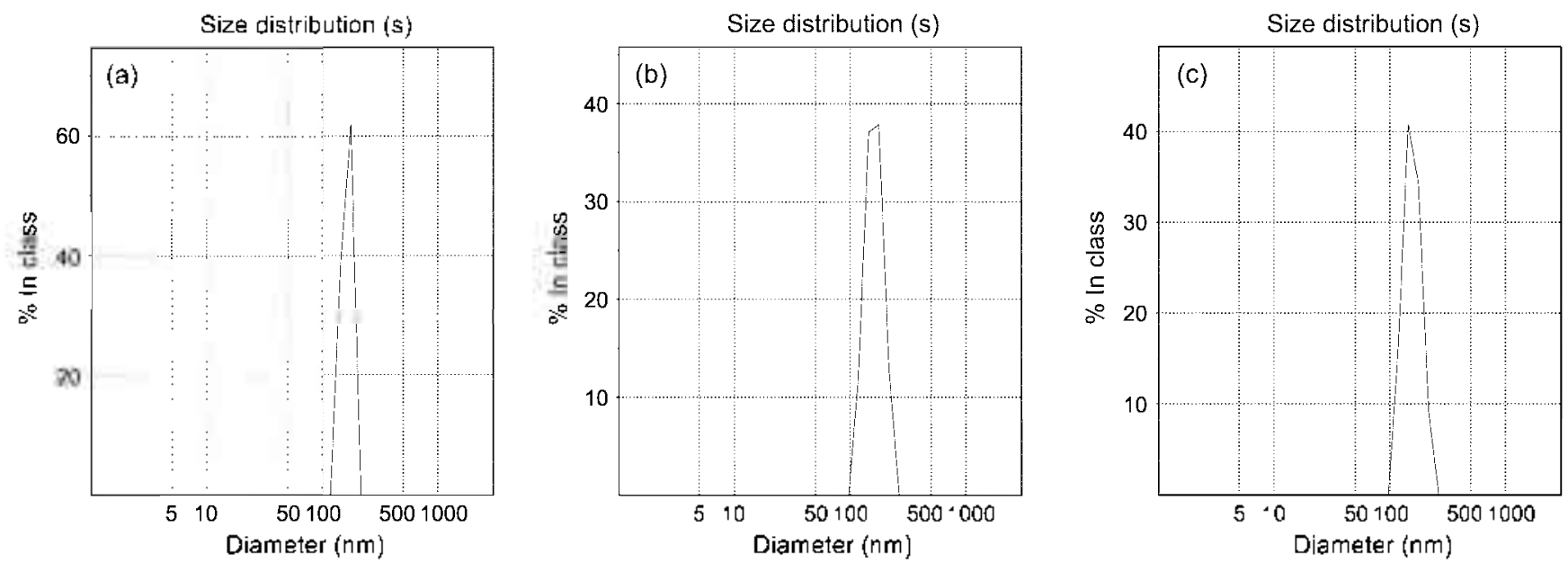

Figure 2. Particle size distribution of the $\mathrm{Fe}_{3} \mathrm{O}_{4}$ nanoparticles (a) size distribution by intensity, (b) size distribution by volume, (c) size distribution by number. 


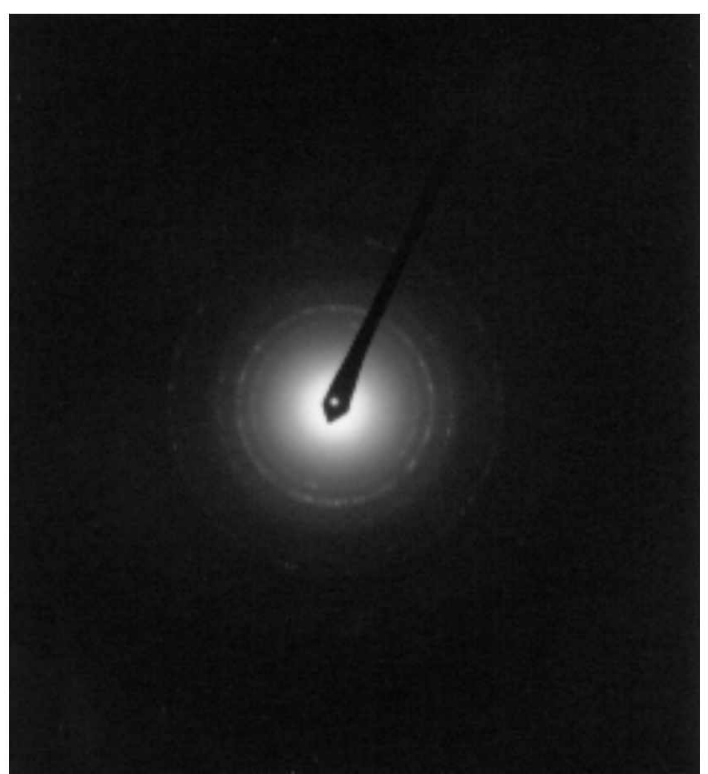

Figure 4. Electron diffraction pattem of $\mathrm{Fe}_{3} \mathrm{O}_{4}$ nanoparticles.

5 shows IR spectra of the magnetite nanoparticles. As shown in Figure 5. the characteristic peak of $\mathrm{Fe}_{3} \mathrm{O}_{4}$ is $570.8 \mathrm{~cm}^{-1}$ and $3400 \mathrm{~cm}^{-1}$ is the hydroxy characteristic peak of water absorbed in the surface. Several absorption bands were observed in the figure. such as at $3423.0 \mathrm{~cm}^{-1}$ is the hydroxy stretching vibration characteristic peak. $2925 \mathrm{~cm}^{-1}$ is stretching vibration characteristic peak of carbon hydrogen and $1015 \mathrm{~cm}^{-1}$ is stretching vibration of carbon oxygen. 1436 $\mathrm{cm}^{-1}$ is bending vibration peak of hydroxy group. The intensity of the band from the $\mathrm{Fe}_{3} \mathrm{O}_{4}$ at decreased relative to the bands of soỵbean lecithin with increasing soỵbean

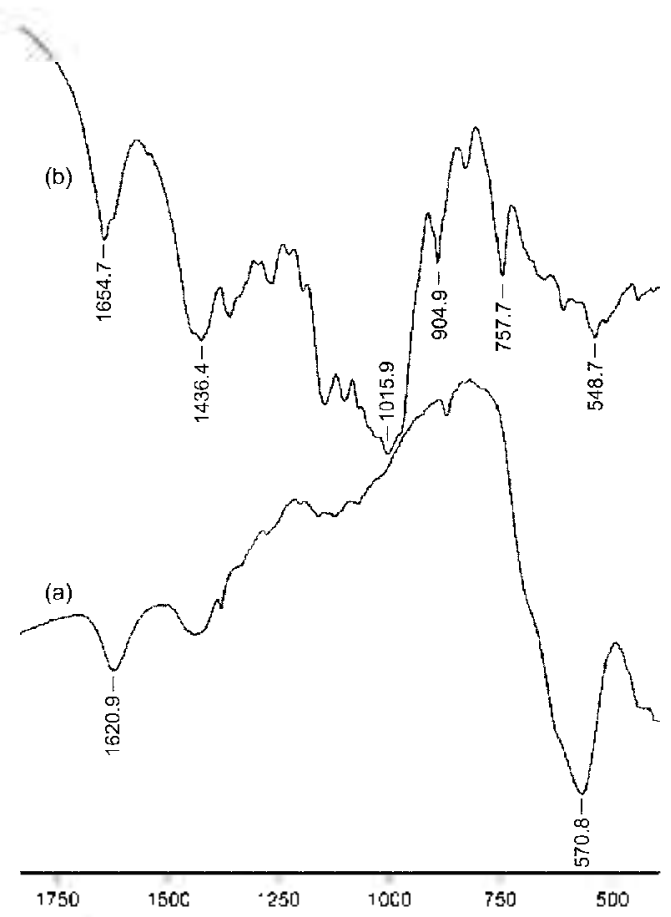

Figure 5. Intrared spectra of the $\mathrm{Fe}_{3} \mathrm{O}_{4}$ nanoparticles (a) coated with the soybean lecithin (b) $\mathrm{Fe}_{3} \mathrm{O}_{4}$. lecithin concentration. these results indicate that soybean lecithin irreversibly adsorbed on the surface of $\mathrm{Fe}_{3} \mathrm{O}_{4}$ particles even after carefully washing.

Soybean lecithin coverage ${ }^{11.14}$. The Thermogravimetric analysis was used to measure soybean lecithin coverage ( $\Gamma$ expressed in $\mu \mathrm{mol} \mathrm{m} \mathrm{m}^{-2}$ ). The adsorbed amount of on the magnetite particles was detenmined by TGA (PerkinElmer TGA-7) scanning from room temperature to 480 at $10^{\circ} \mathrm{C}$ $\mathrm{min}^{-1}$. The chemisorption of the soybean lecithin on magnetite is shown in Figure 6 where the soybean lecithin coverage is plotted against equilibrium concentration, at low total soybean lecithin concentration (less than $=11 \mu \mathrm{mol} / 1$ ). soybean lecithin is mostly adsorbed. At higher soybean lecithin concentration, the saturation level of $0.16 \mu \mathrm{mol} \mathrm{m}^{-2}$ is reached. The isotherms are Langmurian in nature (Fig. 7). The Langmuir analysis of the soybean lecithin coating was performed with the help of equation:

$\mathrm{C}_{\mathrm{eq}} / \Gamma_{\mathrm{L}}=1 / \Gamma_{\mathrm{L}}{ }^{\prime \prime}\left(\mathrm{C}_{\mathrm{eq}}+1 / \mathrm{K}_{\mathrm{L}}\right) . \Gamma_{\mathrm{L}}{ }^{\prime \prime}$ is the value at saturation, $\mathrm{K}_{\mathrm{L}}$ is the association constant. $\mathrm{K}_{\mathrm{L}}$ value was calculated to be $3.01 \mathrm{mM}^{-1}$ (Fig. 6).

Crystallographic and Magnetic Properties. Figure 8 shows the change in the X-ray diffraction patterns. The XRD peaks correspond to the spinnel structure in all the samples. These peaks did not shift but became broader. The broadening of XRD peaks was observed in $2 \theta$ : 30.00 .35 .82 . 43.34. 54.34,57.32. 62.84 etc. The broadening of XRD peaks is therefore predominantly attributed to the small size of crystallite. which is directly related to the decrease in the particle size. We attribute the broadening of XRD peaks to soybean lecithin, which is not removed. which reflect that space distance of the ordered systems fomed is molecular distance. Because the change of the nanoparticles size brings about change of XRD diffraction pattems. we can use Scherrer formula to estimate the particle size. The volume average relative particle size of the crystal. D. was calculated with the Scherrer formula. ${ }^{15}$

$$
\begin{aligned}
& \mathrm{D}=\mathrm{K} \lambda / \beta \cos (\theta) \\
& \beta=\mathrm{B}-\mathrm{b}
\end{aligned}
$$

Wherein: $\mathrm{D}$ : is the mean crystal size $\lambda$ : is the $\mathrm{X}$-ray wavelength (nn), $\theta$ : is the Bragg angle. $\beta$ : is the excess line broadening (radian), $\mathrm{B}$ is the linewidth (radian) and $\mathrm{b}$ is an instrument line broadening (radians), $\mathrm{K}$ : is the constant

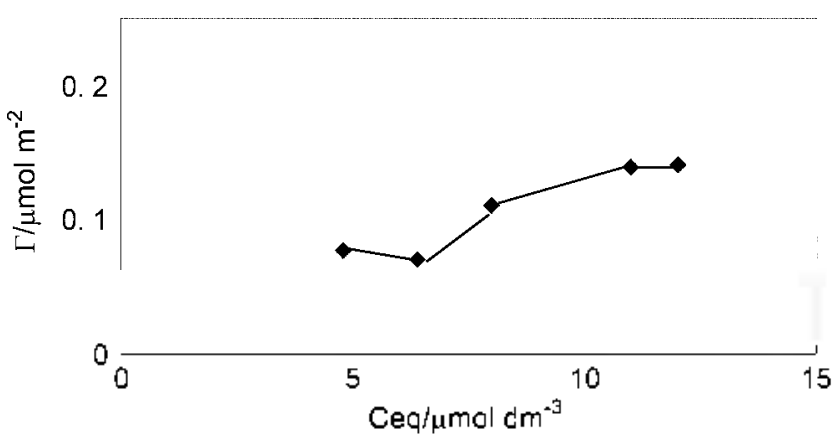

Figure 6. Evolution of the adsorbed amount as a function of total soybean lecithin concentration. 


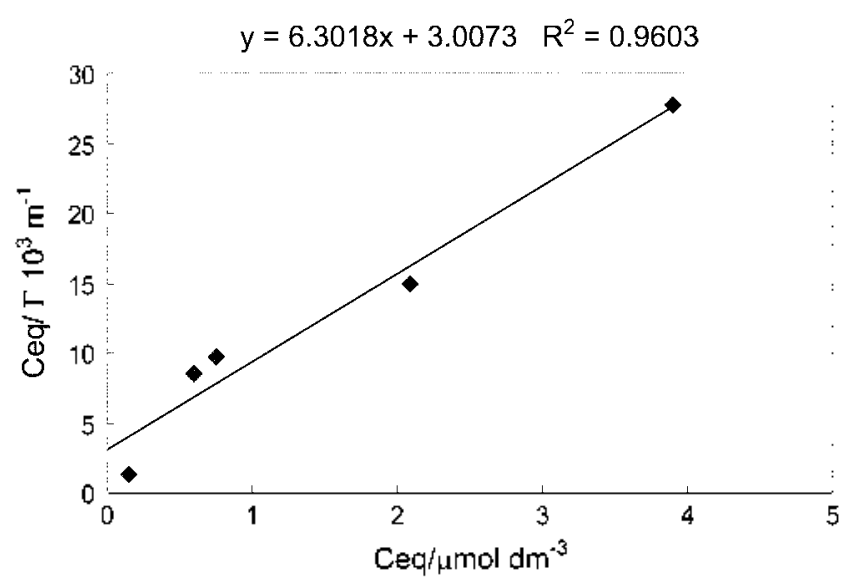

Figure 7. Langmuir plot of sorbean lecithin adsorption (equilibrium free sovbean lecithin/adsorbed sovbean lecithin as a function of equilibrium free soybean lecithin) at micromolar concentration. Upper equation comespond to the Langmuir regression $\mathrm{C}_{\text {ey }} T_{\mathrm{L}}=$ $1 / \Gamma_{\mathrm{L}}^{0}\left(\mathrm{C}_{\mathrm{z} \alpha \mathrm{l}}+1 / \mathrm{K}_{\mathrm{L}}\right) . \Gamma_{\mathrm{L}}{ }^{0}$ is the value at saturation, $\mathrm{K}_{\mathrm{L}}$ is the association constant.

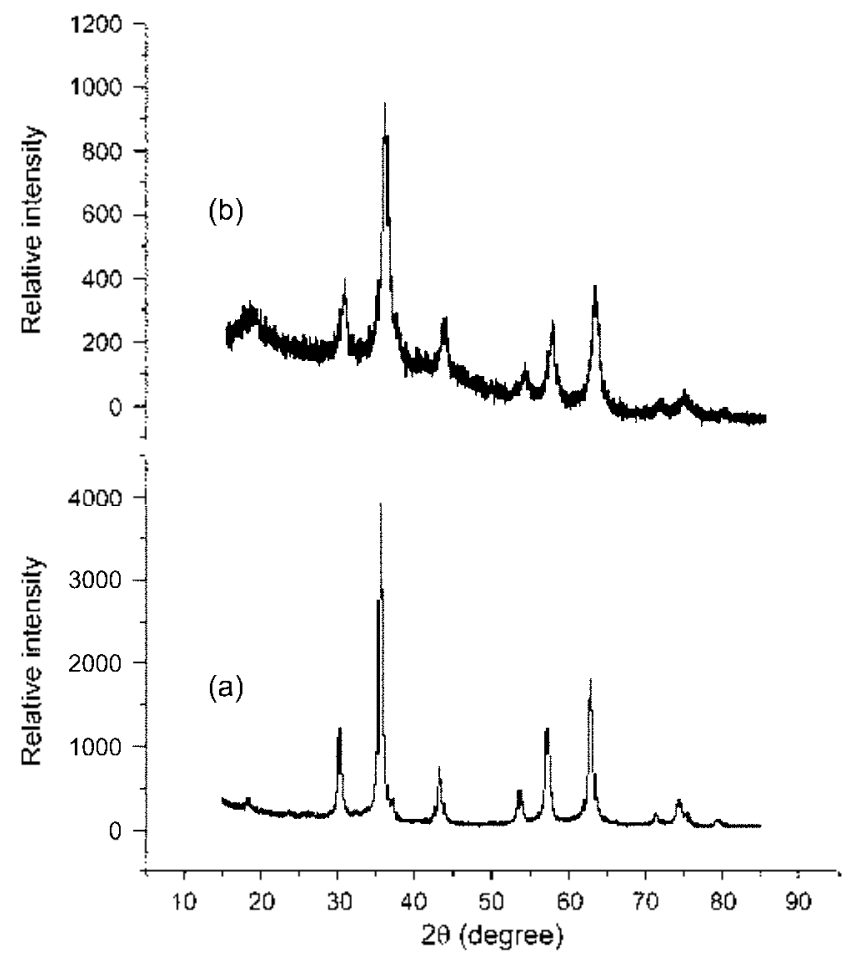

Figure 8. X-ray diffiaction pattern of the $\mathrm{Fe}_{3} \mathrm{O}_{4}$ nanoparticles. (a) $\mathrm{Fe}_{3} \mathrm{O}_{4}$, (b) coated with soybean lecithin. which is approximately equal to unity and is related both to the crystallite shape and to the way in which $\beta$ and $\mathrm{D}$ is define. According to Scherrer formula, the diameter of particle size is about $7 \mathrm{~nm}$, the result is corresponding with TEM. This shows that the soybean lecithin was added to limit the particle size and to form the core of fine particle $\mathrm{Fe}_{3} \mathrm{O}_{4}$.

\section{Conclusion}

Stable magnetite colloidal dispersions with average particle size about $7 \mathrm{~nm}$ has been successfully prepared by coprecipitation in a soybean lecithin aqueous solution. Each particle was covered with a soybean lecithin layer. From the measurements by TEM. The diameter of the $\mathrm{Fe}_{3} \mathrm{O}_{4}$ nanoparticle is about $7 \mathrm{~nm}$. The investigation of the Infrared spectra and $\mathrm{TGA}$ of $\mathrm{Fe}_{3} \mathrm{O}_{4}$ nanoparticle showed that the surfaces were protected by the soybean lecithin. Phase analy sis of the final product by XRD confirmed the formation pure $\mathrm{Fe}_{3} \mathrm{O}_{4}$ nanoparticle.

Acknowledgmments. This work was partly supported by the National Nature Sciences Foundation of China (Grant Number: 39570843). We thank Prof. Desen Su for his helpful discussion.

\section{References}

1. Hayashi, C. Ploys Todav 1987, 10.44

2. Gleiter. H. Prog Hater: Sci. 1989. 33. 223.

3. Karch. H.: Birringer. R.: Gleiter. H. Nature 1987. 330.556.

4. Fendler. T. H. Chent. Ren: 1987. 87. 887.

5. Liu. C.: Rondinone. A. J.: Zhang. Z. J. Pure Appl. Chem. 2000. $72(1-2), 37$.

6. Bonnemain. B. J. of Drug Targeting 1998, 6(3). 167.

7. Pulfer. S. K.: Gallo. T. M. J of Drtug Targeting 1998. 6(3). 215.

8. Illum. L.: Church. A. E.: Butterworth. M. D.: Ariet1. A.: Whetstone. J.: Davis. S. S. Pharnaceutical Rese $a c h$ 2001. 18(5). 640.

9. Denizot. B.; Tanguy, G.: Hindre. F.: Rump. E.; Jacques. J.: Jeune, L. Jallet, P. J. Colloid hnterface Sci. 1999. 209.66

10. Marn. S.; Hannington. J. P. i. Colloid Interface Sci. 1988. 122(2). 326

11. Butterworth. M. D.: Illum. L.: Davis. S. S. Collids \& Surfaces: Phusiochemical \& Engineering Aspects 2001. 179(1).93.

12. Bhandarkar. S.: Bose. A. J. Colloid hnterfoce $S_{c i}$ 1990. 135 (2). 531.

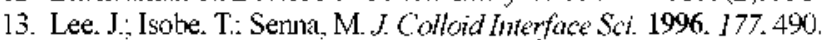

14. Denizot, B.: Tanguy, G.: Hindre. F. et al. J. Colloid Interface Sci. 1999. 209.66

15. Klug. H. P.: Alesander. L. E. M-Rav Diffiaction Procedures for Polyoystalline and Amorphous Materials: Wiley: New York. 1967. 